\title{
The mirror of physics: on how the Price equation can unify evolutionary biology
}

\author{
Victor J. Luque ${ }^{1}$ (D) $\cdot$ Lorenzo Baravalle $^{2}$
}

Received: 30 September 2020 / Accepted: 26 July 2021 / Published online: 5 August 2021

(c) The Author(s) 2021

\begin{abstract}
Due to its high degree of complexity and its historical nature, evolutionary biology has been traditionally portrayed as a messy science. According to the supporters of such a view, evolutionary biology would be unable to formulate laws and robust theories, instead just delivering coherent narratives and local models. In this article, our aim is to challenge this view by showing how the Price equation can work as the core of a general theoretical framework for evolutionary phenomena. To support this claim, we outline some unnoticed structural similarities between physical theories (in particular, classical mechanics) and evolutionary biology. More specifically, we shall argue that the Price equation, in the same way as fundamental formalisms in physics, can serve as a heuristic principle to formulate and systematise different theories and models in evolutionary biology.
\end{abstract}

Keywords Evolutionary biology · Price equation · Complexity · Metatheoretical structuralism $\cdot$ Guiding principle $\cdot$ Classical mechanics

This article belongs to the topical collection "Simplicity out of Complexity? Physics and the Aims of Science," Edited by Florian J. Boge, Martin King, Paul Grünke and Miguel Ángel Carretero Sahuquillo.

Victor J. Luque

victor.luque@uv.es

Lorenzo Baravalle

lbaravalle@fc.ul.pt

1 Department of Philosophy, University of Valencia, Avda. Blasco Ibáñez, 30 - Planta Setena, 46010 Valencia, Spain

2 Centro de Filosofia das Ciências, Departamento de História e Filosofia das Ciências, Faculdade de Ciências, Universidade de Lisboa, Campo Grande; Edifício C4, $3^{\circ}$ Piso, Sala 4.3.24,

1749-016 Lisboa, Portugal 


\section{Introduction}

Advanced areas in science, especially physics, have developed sophisticated mathematical apparatus in which specific formulas such as Newton's second law of motion, Einstein's equations of special and general relativity, Schrödinger's equation, etc., play the role of fundamental equations. These fundamental equations provide, among other things, unified patterns of explanation for the phenomena under study. Nevertheless, most evolutionary biologists and philosophers of biology are sceptical about the possibility of attaining similar formalisms in evolutionary biology. Due to its high degree of complexity and its historical nature (Bartholomew, 1986), evolutionary biology is usually considered a messy science (Tawfik, 2010). Even Ronald Fisher, who is typically considered as a supporter of the view according to which physics and biology share some important similarities, in 1932 claimed:

I believe that no one who is familiar, either with mathematical advances in other fields, or with the range of special biological conditions to be considered, would ever conceive that everything could be summed up in a single mathematical formula, however complex. (Fisher, 1932, p. 166).

A common view in evolutionary biology may be summarised as follows: biological evolutionary systems are too complex to be captured by an austere, general and encompassing formalism, thus we need to focus on narrow theories and specific models. This view is nowadays strictly linked to a distrust concerning the explanatory power of the theoretical framework developed within the so-called "Modern Synthesis". Over the last hundred years, evolutionary biology has been extremely successful in explaining a wide range of evolutionary phenomena. A great deal of this success can be credited to the fact that biologists like Ronald Fisher, J.B.S. Haldane, and Sewall Wright developed a mathematical theory of evolution-i.e., population genetics-integrating Darwin's ideas on natural selection with Mendelian genetics. However, starting from the 1960s, the apparent consensus over the Modern Synthesis began to be eroded (Mitchell \& Dietrich, 2006). More recently, several researchers have called for an Extended Evolutionary Synthesis for evolutionary biology (Laland et al., 2015; Pigliucci \& Müller, 2010), stressing the importance of different and diverse mechanisms and causes in evolution-such as niche construction, macroevolution, gene networks, or nongenetic inheritance-which cannot easily be accounted for through traditional population genetics.

The very ambition for a general and unified theory of evolution has frequently been labelled simply as a "physics envy" (Egler, 1986; Lockwood, 2007; Mayr, 2004), and this kind of theory has been considered as unattainable for evolutionary biology. A related idea is that, due to the theoretical differences between physics and evolutionary biology, we should not waste our time seeking general principles. Instead, we should accept the particularity of evolutionary biology and only focus on local and circumstantiated models of evolution (Mitchell \& Dietrich, 2006; Pigliucci, 2002; Waters, 2011). Some of the authors arguing for such a "fragmentalist" view selectively focus on the aspects that distinguish physics from evolutionary biology, ignoring other features that are possibly common to the two disciplines. 
In our view, one of the reasons for this is that such authors implicitly ground part of their understanding of what a scientific theory is on an outdated philosophy of science. Common prejudices, in this sense, are that a scientific theory must provide "universal unrestricted generalisations" (e.g., Hempel, 1965) or that highly unified theories are constituted by sets of laws deductively connected (e.g., Carnap, 1995). Since evolutionary biology, unlike physics, is unable to attain such standards, then it would be unable to provide well-defined and general theoretical frameworks. While some authors (e.g., Fodor \& Piattelli-Palmarini, 2010; Popper, 1974) take this as evidence that evolutionary biology is something like an "inferior science", others interpret it as evidence that science does not require the formulation or identification of laws (Beatty, 1995; Lockwood, 2007; Mayr, 2004; O’Hara, 2005; Pigliucci, 2002).

We think that the perspective dismissive of the life sciences is largely unwarranted. The great complexity of the phenomena that evolutionary biology is called on to explain does not imply that they cannot be tamed by some unificatory conceptual apparatus similar to those developed within physics. The historical nature of biological evolutionary processes is not necessarily at odds with an overall formal approach to evolutionary dynamics. Recent works on the Price equation are shedding new light on the possibility of conceiving evolutionary biology as a highly unified field. The Price equation has been proposed as the fundamental equation of evolution (Lehtonen, 2018; Luque, 2017; Queller, 2017; Rice, 2004) because, among other reasons, it requires fewer assumptions than other equations of evolutionary biology (e.g., Fisher's fundamental theorem or the breeder's equation) which have been historically considered as fundamental. In addition, these fundamental equations can easily be derived from the Price equation with additional assumptions. Our goal in this article is to clarify in which way the Price equation may contribute to changing our view about the supposed peculiarities of evolutionary biology with respect to other sciences.

To this aim, we shall use the following argumentative strategy. First of all, in Sect. 2, we shall introduce some notions derived from metatheoretical structuralism (Balzer et al., 1987) concerning the structure of scientific theories. As a paradigmatic example of a well-structured scientific theory, we shall discuss Newtonian mechanics. In particular, we shall emphasise the role of Newton's second law as the fundamental law of this theory. In Sect. 3, we shall outline the conceptual framework behind the Price equation. We shall then argue that the Price equation satisfies the structuralist criteria for a fundamental law. At the same time, we shall show that the genetic theory of evolution can indeed be represented as a well-structured theory, unified under the Price equation. The goal of this discussion is to show that there are not, at least in principle, structural differences between a theory in physics and a theory in evolutionary biology. A supporter of the fragmentalist view might easily relativise this claim by arguing that, while Newtonian mechanics was once considered to account for any physical phenomenon, the genetic theory of evolution (that is, the "good old-fashioned" population genetics) is just a small part of evolutionary biology. A fully satisfactory theory of evolution should be able to account for all the diverse and complex phenomena which are not properly explained through traditional population genetics. In Sect. 4, we shall show that the Price equation can be 
reformulated and applied outside genetic evolution to explain a broad range of "nonclassic" evolutionary dynamics. This will allow us to emphasise, in Sect. 5, another sense in which evolutionary biology resemble physics.

\section{Theories, laws and unification in metatheoretical structuralism}

Metatheoretical structuralism (Balzer et al., 1987) is a semantic approach to the reconstruction of the structure of scientific theories. Over the last 30 years or so, it has been employed to elucidate the structure of many different scientific theories in physics (classical and relativistic mechanics, thermodynamics, etc.), biochemistry, biology (Darwinian theory, Mendelian genetics, population genetics, etc.) economics and sociology (see Diederich et al., 1994; Díez \& Lorenzano, 2002 for overviews).

One of the tenets of metatheoretical structuralism is that the term "scientific theory" is intrinsically polysemic, that is, it admits many characterisations (see, for instance, Moulines, 2010). Sometimes, we talk about theories in quite a strict sense, to denote the core of a mature theory. This is, for instance, the case when we present-frequently for pedagogical reasons-Newtonian mechanics as the set composed of Newton's three laws of motion and the law of universal gravitation. This narrow characterisation of the notion of theory places in the foreground generalisations which are considered, both conceptually and historically, as fundamental for the development of a scientific field. These generalisations roughly correspond to what old-fashioned syntactic conceptions of scientific theories (e.g., Carnap, 1995; Feigl, 1970) considered the axioms of the theory. One of the reasons for the obsolescence of these conceptions, nonetheless, is precisely that in a scientific theory there is far more than its axioms. In a broader, and more proper sense, a theory, in fact, also includes many other more specific laws with a more restricted domain of application. In Newtonian mechanics, this broader set of laws includes Hooke's law, the laws of pendulum, etc. Moreover, since theories in this broader sense are historical entities, when characterising them we must also take into account their possible reformulations (such as Lagrangian and Hamiltonian mechanics in the case of Newtonian mechanics) and the changes in their intended domain of application.

To make sense of this complex picture, metatheoretical structuralists give up the logical empiricist ideal that scientific theories have an axiomatic-deductive structure, characterisable in purely syntactic terms. Instead, they represent theories-in the broader sense-as collections of models (in the sense of mathematical model theory) called theoretical elements, which are connected by an antisymmetric and transitive relation of specialisation. Each theoretical element roughly corresponds to a law of the theory and identifies, so to speak, a portion of the world that works according to that law. ${ }^{1}$ By adopting this characterisation, structuralists also give up

\footnotetext{
1 The theoretical elements of a theory are usually analysed, in metatheoretical structuralism, as set-theoretical predicates. This analysis permits a formally rigorous characterisation of the constitutive features of the laws of a theory (such as its intended scope, the theoreticity of the concepts occurring in the law, etc.). We need not enter into such a fine-grained level of analysis here (the interested reader is referred to Balzer et al., 1987).
} 
the logical empiricist notion of law as a "universal unrestricted generalisation". For metatheoretical structuralism, as for most semantic approaches, nomicity does not necessarily imply universality, but rather counterfactual support. Counterfactual support does not require universality because a statement can offer counterfactual support even when it is not intended to account for a wide range of phenomena, but is limited in its scope (for more details about this, see Lorenzano, 2006; Lorenzano \& Díaz, 2020).

In mature theories, theoretical elements can profitably be arranged in the form of an inverted tree called a theory-net. At the top of a theory-net we usually find a single theoretical element, including some very general assertion about the way in which the theory accounts for a class of phenomena: this is the fundamental law. The specialisations specify regularities concerning subclasses of the class of phenomena described by the fundamental law. The relation of specialisation is thus understood as restricting the scope of application of the fundamental law. Through its specialisations, a theory can provide the details of the behaviour of specific parts of the world which it is intended to explain. A theory-net can change its configuration through the history of the theory, depending on the discovery/formulation of new specialisations and/or the broadening or the narrowing of the domain of application of the theory.

This is (Fig. 1) a possible structuralist reconstruction of the overall structure of Newtonian mechanics in a specific phase of its historical development:

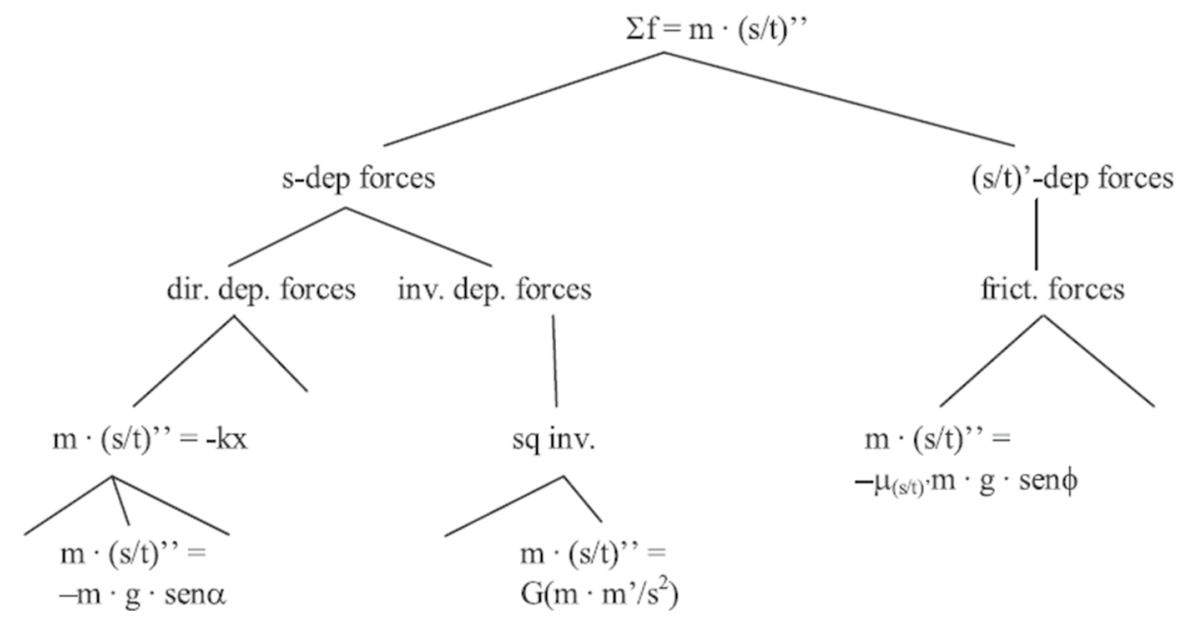

Fig. 1 Structuralist reconstruction of Newtonian mechanics as a theory-net (from Díez \& Lorenzano, 2013, p. 1161). The specialisations of Newton's second law are hierarchically represented as follows: the first branching distinguishes between space-dependent and velocity dependent forces; the space-dependent forces branch into direct and indirect space-dependent forces; direct space-dependent forces branch into linear negative space-dependent forces and others; and so forth 
As we have just said, the laws of a theory do not usually gain their explanatory or predictive role from their generality, but from the fact that they identify invariant relations of counterfactual dependence between certain properties of the phenomena under study. An important exception to this is the fundamental law, which in the case of Newtonian mechanics is Newton's second law. According to structuralists, this law, taken individually, is "almost (empirically) vacuous": it does little more than provide a definition for the notion of force (Díez \& Lorenzano, 2013). Its domain of application is almost entirely specified by the other theoretical elements of the theory-such as the Newton's third law, the law of gravitation, Hooke's law, etc.- - which are its specialisations. So, why is Newton's second law so important? Because it is the guiding principle of Newtonian mechanics (Moulines, 1984a). The fundamental law of a theory establishes "what the theory is about" by providing a very general and comprehensive schema for explaining a class of phenomena. Newton's second law states a condition of possibility for something to be considered as a physical force (Baravalle \& Vecchi, 2020; Jammer, 1956). It inspired generations of physicists, by pointing out "where they had to look" in order to explain the behaviour of the physical world. The structuring role provided by the fundamental law of a theory with respect to its development is thus, in the structuralist conception, mostly heuristic. It is not an "axiom" from which all the other laws of the theory must be derived, but a general assertion providing a shared (formal) vocabulary for the practitioners in a field (Lorenzano, 2006).

Besides providing heuristic guidance, fundamental laws have-according to methateoretical structuralists (see, for instance, Moulines, 1991; Lorenzano, 2006) some other interesting properties. These properties are: (1) a synoptic character; (2) applicability to all the models of the theory; (3) empirical unrestrictedness; (4) a systematiser role. Concerning the first property, fundamental laws have a synoptic character insofar as they include all the fundamental concepts of the theory. These are the concepts that occur-albeit in some different form-in any specialisation of the theory. The second property - that is, its applicability to all the models of the theory-is especially important because it is linked to the generality of fundamental laws. Regarding the third property, to say that fundamental laws are empirically unrestricted is similar to saying that, as we have already seen, they are "almost vacuous". The phrase "empirical unrestrictedness", however, better emphasises the fact that-differently from empirical generalisations-fundamental laws are somehow "a priori" and "constitutive" (see Friedman, 2001 for an analogous, non-structuralist, view). Finally, fundamental laws play a systematiser role in the sense that they shape the theory, by pointing out a natural way to develop its structural features. Newton's second law, of course, has all these properties. It contains all the fundamental concepts (force, mass, acceleration) of classical mechanics - that is, it has a synoptic character. The fact that all the specialisations of the theory are, so to say, attempts to empirically characterise Newtonian forces also reveals that Newton's second lawwhich exhibit what all Newtonian forces have in common-applies to any possible model of the theory. This generality is attained, among other things, thanks to the empirically unrestricted character of Newton's second law, which-rather than describing any real-world phenomenon-states a condition of possibility for something to be considered as a "Newtonian force". Finally, and in virtue of the previous 
three properties, Newton's second law shaped classical mechanics insofar as it determined an agenda for physicists.

To this sense of the notion of theory-i.e., theory as a theory-net-we may finally add a last, perhaps vaguer, one. Historically, scientific theories do not develop in a disciplinary vacuum: laws and scientific concepts elaborated with the aim of explaining a certain domain of phenomena are frequently adopted by scientists working in other fields and adapted to account for other phenomena. Take again, as example, the case of Newtonian mechanics: although initially intended to explain phenomena related to planetary and terrestrial motion, it was progressively extended to explain other physical phenomena (such as fluid or gas dynamics, in hydrodynamics and thermodynamics respectively) and some of its fundamental concepts have been consistently adopted in other theories (e.g., the concept of force appears both in Newtonian mechanics and in Lorentz's equation in electrodynamics; Moulines, 1984b). Furthermore, Newtonian mechanics has been adopted as a general framework to understand phenomena outside the domain of physics (Darwin himself regarded Newtonian mechanics as a model for his theory; e.g., Depew \& Weber, 1996). In other words, we may say that theories (intended as theory-nets) can establish intertheoretical links with other theories-by exchanging with them concepts and nomological generalisations - and, thus, generate "families of theories", which structuralists call theoretical holons (Balzer et al., 1987, Ch. 8).

We shall say something more on theoretical holons in Sect. 5. For the moment, and before proceeding to consider how these ideas can be applied to the genetic theory of evolution and the Price equation, let us consider some important lessons emerging from the structuralist conception of scientific theories and laws.

First of all, when we assess the "generality", or the "unificatory power", of a theory we need to be clear about the level at which we are examining the theory. Many laws in physics are not "universal unrestricted generalisations" (think, for instance, about Kepler's law or Galileo's law of free-fall in classical mechanics). Only the fundamental law is required to be "universal" in the sense that it provides a general "explanatory pattern" (à la Kitcher, 1989). The components of a theory play very different roles within a theory: while it would be absurd to ask of a fundamental law that it provide any detail about specific features of the physical world, it would be equally unrealistic to ask of the specialisations of the theory that they be of universal application. This is because what we want from a fundamental law is that it unifies a certain class of phenomena under a very comprehensive description, whereas the specialisations are intended to empirically adapt such a description to explain specific phenomena. To complain that the specialisations of a theory are not general enough is tantamount to not having understood their role in the theory. Some specialisations, of course, will be more encompassing (in terms of their possible applications) than others, but generality is not the fundamental feature of a specialisation. This should be taken into account when it is sometimes loosely said that evolutionary models are "local" or of "limited scope" (van Veleen, 2005; Waters, 2011). In a sense, this is not something specific of evolutionary models, but common to any non-fundamental law in science.

Possibly even more important to notice here is that, as long as there are at least three senses in which we can talk of "scientific theories", we should be more careful 
when we say things such as "physical theories are more unified than biological theories", or "physical theories are grounded on encompassing formalisms, while biological theories are not". This may be true if we consider a "theory" in a certain sense, but false if we consider it in another sense. We suspect that when the supporters of the fragmentalist view say that evolutionary biology is too messy to be able to attain a unitary and cohesive form, like the one displayed by physical theories, they are incorrectly comparing physical theories qua theory-nets and evolutionary biology qua a theoretical holon. As we have seen, theory-nets are hierarchically organised under a guiding-principle, while theoretical holons are more disperse families of theories sharing concepts (they perhaps identify "scientific disciplines" as a whole). Thus, of course a theory-net is more "unified" than a theoretical holon. The right thing to do, in order to fairly compare physics with evolutionary biology, would be first to check if it is possible to identify theory-nets in evolutionary biology. This has been already done for the classic theory of natural selection (Díez \& Lorenzano, 2013; Ginnobili, 2018) and traditional population genetics (Lorenzano, 2014). In the next section, we shall argue that the genetic theory of evolution can be properly depicted as a theorynet, unified under a certain version of the Price equation. This would show, in our opinion, that there is no conceptual impediment, in evolutionary biology, to formulating theories which are structurally analogous to physical ones. We agree on the fact that these theories are somehow "specific", in the sense that they are unable to account for all evolutionary phenomena. But, in a certain sense, the same happens in physics: neither Newtonian mechanics, nor thermodynamics or general relativity account for all physical phenomena. This kind of comprehensiveness is only attained at the level of theoretical holons: the family of all physical theories. If we look at that level, however, we again see similarities between physics and evolutionary biology: both disciplines are, to a certain extent, a patchwork of many theory-nets, connected in a vaguer way by some fundamental concepts. Concerning this level of analysis, we shall suggest in Sect. 5 that, once more, the Price equation plays an important role in coordinating different theories within evolutionary biology.

\section{The Price equation and the genetic theory of evolution}

In the early 1970s, George Price $(1970,1972)$ presented an abstract formula to represent evolutionary change. Because of the lack of substantive biological assumptions, it is usually stated the Price equation does not involve idealisations (GodfreySmith, 2009) or that it is an assumption-free statement about evolution (Walsh \& Lynch, 2018). To be precise, the Price equation does actually rely on some minimal assumptions, but they are not simplifying ones. They are instead intended to establish the basic properties of any evolutionary system. ${ }^{2}$ It is worthwhile, in this respect, to compare the traditional approach in population genetics and the Pricean

\footnotetext{
2 Some additional assumptions made by George Price when he derived the original Price equation (such as the conceptualisation of a population as a closed system) are less fundamental, and therefore can be relaxed in order to incorporate more complex scenarios (Godfrey-Smith 2009). See also footnote 6.
} 
approach. The former starts deliberately with idealised assumptions of the target systems, producing simplified models. The Pricean approach, in contrast, starts with postulates or assumptions that we think are true about the target system (what the properties of the system are) and we then derive its mathematical rules (Rice, 2004, 2020; Luque, 2017). ${ }^{3}$

These basic properties of evolutionary systems, according to the Pricean approach, can be summarised as follows (Rice, 2004, p. 169):

- Change over time To be considered as evolutionary, a system requires a population of characters changing over generations. In order to represent generations, we may use any time interval that is appropriate for the system under study.

- Ancestor/Descendant relations Different generations of an evolving population must be connected by some character. This is the basic requirement for inheritance.

- Character or phenotype Any feature of an entity that can be represented with a real number is, potentially, a phenotypic character. Accordingly, very different entities - such as alleles, genotypes, groups of individuals, etc.-can be interpreted as endowed with evolving characters.

Although sometimes presented with different notation, the Price equation is usually written as

$$
\bar{w} \Delta \bar{z}=\operatorname{Cov}(w, z)+E(w \Delta z)
$$

where $\Delta \bar{z}$ is the change in average value of a character $z$ (in this case its mean, but it can also be adapted to represent higher moments such as variance, skew, etc.) over an arbitrary time step (usually one generation), $w$ is the absolute fitness of an individual (calculated as the number of descendants), and $\bar{w}$ is the average fitness. The first term on the right-hand side is the covariance between fitness $w$ and the character $z$, and represents the change in the average value of $z$ due to differential survival and reproduction. Note that, since this term is just a measure of the statistical association between the character and fitness, it does not necessarily represent a causal connection between them. For this reason, although it is typically interpreted as representing natural selection, it represents the effects of drift as well (Rice, 2004). The second term on the right-hand side is the expected value of the quantity $\Delta z$, which measures how much the offspring deviate from their parents with respect to the character $z$ (i.e., it accounts for the change in the value of the character from the parents to the offspring due to processes involved in reproduction). Thus, the second term represents the fidelity of the transmission of the organisms with respect to the

\footnotetext{
3 Sean Rice characterises this procedure as axiomatic: "A goal of the axiomatic method in mathematics is to start out with a minimal set of necessary axioms and then derive further results from these. The analogue in science would be to start out with a minimal set of scientific axioms and ask how far we can get deriving new results from these before we have to introduce simplifying assumptions" (Rice 2020, p. 1).
} 
character $z$. If the fidelity of the transmission is not perfect, then some evolutionary forces such as mutation, recombination, selection at a lower level of organisation, or other processes, are causing a transmission bias.

Arguably, the Price equation has all the properties that structuralists ascribe to fundamental laws in science. ${ }^{4}$ First, it contains all the fundamental concepts in the description of evolutionary dynamics, that is, it has a synoptic character. One may in fact say that the most important result that George Price obtained in his 1970's derivation of his equation was the identification of the basic ingredients of any evolutionary process, as well as of a procedure to formalise them. To many people, these ingredients and this procedure may now seem "obvious" or "trivial". But evolutionary biology had to wait until the arrival of George Price to have a clear grasp of them. ${ }^{5}$ Any model in population genetics can be interpreted as specifying a particular mode of evolution, in which the change over time of a character is determined by a certain ancestor/descendant relation in a concrete ecological scenario. This satisfies the second structuralist criteria for fundamental laws, that is, its applicability to all the models of the theory. The Price equation can be employed, in order to account for specific modes of evolution, by adopting further assumptions concerning more circumscribed scenarios.

Within traditional population genetics, the relation between the Price equation and the other nodes of the theory-net (i.e., the models accounting for the effects of specific evolutionary forces, like selection, drift, etc.) should be considered as follows: the Price equation offers a mathematical partition between two possible kinds of evolutionary change; the change produced by selection, or other sorting/sampling forces (such as drift), which modify the distribution of a trait; and the change produced by transmission biases, which modify the trait itself (by modifying some of its characteristic features; e.g., mutation). There is no evolutionary change without these forces acting on a population of traits and, at the same time, there are no other forces acting within the evolutionary process besides them ${ }^{6}$ (see Fig. 2 for a tentative

\footnotetext{
4 A reviewer pointed out that it may be argued that the Hardy-Weinberg law is more fundamental than the Price equation, in the same way that the law of inertia would be more fundamental than Newton's second law. We disagree with that position. First, the Hardy-Weinberg law only applies to diploid sexual organisms, so it lacks the kind of generality that a fundamental law must have. Second, the Hardy-Weinberg law applies only on the assumption of particulate inheritance, while the Price equation can account for hypothetical evolutionary systems with blending inheritance as well (see Gardner 2011). That is, the Hardy-Weinberg law limitedly applies to an actual, historically accidental, scenario (i.e., the evolution of diploid sexual populations with particulate inheritance), while the Price equation applies more broadly to any possible evolutionary scenario. Third, from the structuralist point of view that we adopt in this article, it can be shown that the Hardy-Weinberg law is just a specialisation of the Price equation, which is obtained when all the terms in the Price equation are assumed to be equal to zero (analogously, the law of inertia is a specialisation of Newton's second law in which acceleration is taken to be equal to zero; Balzer et al., 1987; Lorenzano 2014).

5 In Steven Frank's words: "It is always difficult, in retrospect, to see the originality and insight of a simple idea" (Frank 1995, p. 381).

6 The only possible exception is migration. The original Price equation assumes a closed population. Although the equation can track migration between subpopulations (Frank 1986), it can't take into account migration from outside of the entire population (or meta-population). Still, the equation can be expanded, including an extra term, in order to model an open population (Kerr and Godfrey-Smith 2009).
} 


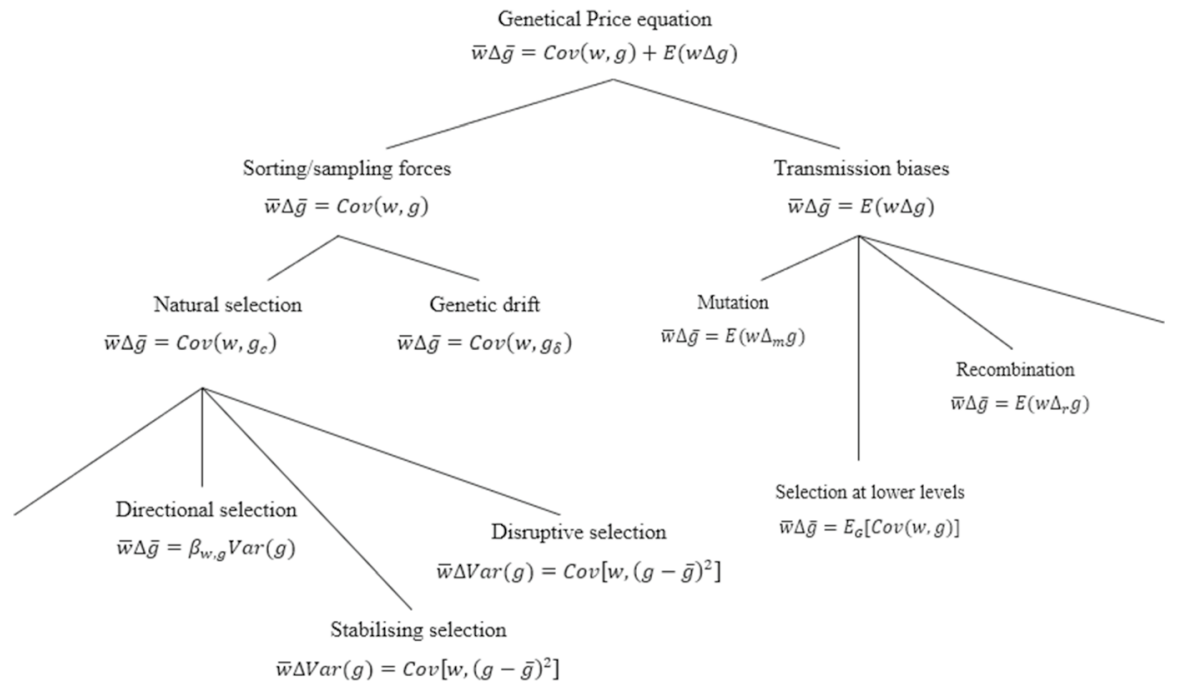

Fig. 2 Tentative reconstruction of the theory-net of Population Genetics. Some branches are left open to stress that it is not a static representation, but a sketch of a theory that is still in development. Equations for natural selection and genetic drift are identical except for the fact that the former requires a causal connection between the trait and fitness, while the latter doesn't. This causal connection is represented by a "c" in $g_{c}$. Also stabilising and disruptive selection equations are identical. If $\operatorname{Cov}\left[w,(g-\bar{g})^{2}\right]<0$, then there is stabilising selection. If $\operatorname{Cov}\left[w,(g-\bar{g})^{2}\right]>0$, then there is disruptive selection. Finally, selection at lower levels is pictured in the transmission bias branch due to the fact that it is derived as a covariance within the transmission term

graphical representation). While the Price equation describes the net change in the diffusion of a trait as the product, or effect, of forces, the specialisations of the theory provide causal characterisations of each force, and these characterisations allow, in their turn, empirical descriptions of the processes acting in evolutionary change. Of course, what concretely counts as a specific sorting factor or transmission bias in a real-world scenario may be different from what counts as a sorting factor or transmission bias in another scenario. This may be considered as a difference between population genetics and Newtonian mechanics, where the specialisations of the theory are usually considered themselves as laws whose applicability is not conditioned by contextual factors. However, this difference is not fundamental once we accept that what the specialisations of a theory do (regardless of if they are usually called "laws" or not) is to identify invariant conditions under counterfactual circumstances, rather than universal regularities. ${ }^{7}$

To continue with how the Price equation satisfies structuralism's requirements for fundamental laws, we can appreciate that the equation is empirically unrestricted.

\footnotetext{
7 At any rate, note that even some Newtonian forces such as elastic and friction forces, and their respective laws (Hooke's law, Amontons' law, Coulomb's law of friction, etc.), are conditioned by contextual factors (see Hitchcock and Velasco 2014).
} 
As a matter of fact, if considered without its specialisations, the Price equation says very little about concrete scenarios. This is an aspect that has led some authors to equate the Price equation to a kind of mathematical tautology (e.g., Nowak \& Highfield, 2011; van Veelen, 2005), devoid of any empirical significance. Instead of seeing this as a defect, we believe (in line with Frank, 2012, Luque, 2017, and our previous discussion in Sect. 2) that this is a strength of the Price equation. A fundamental law does not need, in itself, to be committed to any specific empirical claim concerning the causes of the phenomena to be explained by the theory. Rather, it must provide the heuristics that practitioners must follow in order to succeed in providing the details of the theory. The Price equation is well suited to attaining this goal because it promotes a systematic approach to the categorisation and discovery of the possible causes of evolutionary change (note that this is precisely what is required by the fourth structuralist requirement for fundamental laws).

First of all, the Price equation helps in recovering old results in population and quantitative genetics and clarifying the connections between them. For example, if we assume that $g$ represents the breeding values for a trait, then the "natural selection equation" yields Robertson's theorem, which measures the additive covariance between fitness and the trait, $\bar{w} \Delta \bar{g}=\operatorname{Cov}\left(A_{w}, A_{g}\right)$. This procedure has been applied to many important equations in evolution, such as Fisher's fundamental theorem, the breeder's equation, mutation and recombination equations for haploid and diploid models, etc. ${ }^{8}$ In addition, several new equations and models can and have been derived from the original Price equation, most notably in social evolution (such as several variants of Hamilton's rule and the Multilevel Price equation, Lehtonen, 2020; Marshall, 2015). Moreover, as we shall see in the next section, new "Pricelike equations"--derived by adopting Price's approach to evolutionary dynamics, but not necessarily its original equation-have been formulated outside the traditional boundaries of population and quantitative genetics, to explain a wide range of phenomena.

\section{Complexity in evolutionary systems}

As we mentioned in the Introduction, part of the scepticism concerning the possibility of attaining a well-defined and encompassing "theory of evolution" is due to the fact that evolutionary systems are far more complex than most of the architects of population genetics and the Modern Synthesis seemed to believe. Thus, some researchers have called for an extension of the Modern Synthesis, insisting that evolutionary biology is also called on to explain phenomena such as macroevolution, non-genetic inheritance and developmental constraints on evolution (among others). If we accept the evolutionary relevance of all these phenomena, we must also recognise that traditional models in population genetics cannot easily and systematically account for them. This complexity has required the development of different

\footnotetext{
${ }^{8}$ Due to space limitations we will not show this. See Frank and Slatkin 1990; Rice 2004; Frank 2012; Queller 2017; Lehtonen 2018, for full mathematical details.
} 
theories and models, reinforcing the idea that a unifying framework connecting all of them is doomed to failure.

In this section we shall review evidence that, in spite of the above, researchers have used the Price equation to deal with previously unnoticed aspects of evolutionary systems in a quite effective way. First, in Sect. 4.1, we shall show how the Price equation helps evolutionary biologists to control the explosion of complexity related to the consideration of structural features of populations, and to model specific aspects of evolutionary ecology dynamics in a reasonably straightforward way. In Sect. 4.2, we shall generalise our point, by showing how Price-like equations are proliferating in many areas of evolutionary biology to account for multiple evolutionary causes acting on natural populations - thus contributing to explain many of the neglected phenomena on which the critics of the Modern Synthesis insist.

\subsection{A case study: age-structured populations in evolutionary ecology}

A usual simplifying assumption in population genetics is to think of the target system as a discrete population-i.e., all individuals are born at the same time, reproduce at the same time, and die at the same time. To follow the evolutionary trajectory, and especially the intensity of selection, in this kind of populations, researchers use classical formalisms such as the breeder's equation (or its multivariate form, the Lande equation; Walsh \& Lynch, 2018). Although the evolution of some insect and plant populations can be indeed accommodated within this simplified framework, most other species display overlapping populations, in which individuals coexist with their offspring, mate multiple times, and so on. ${ }^{9}$ In this more realistic and complex situation, the population can be structured by taking into account the distribution of ages of the individuals at a given time, obtaining an age-structured population. ${ }^{10}$ In this kind of scenario, the probability of survival and reproduction of the individuals in a population depends on their age, which affects different components of their fitness. In fact, different parts of a life cycle are correlated with different fitness components such as survival, mating success, fecundity, etc. This phenomenon has been approached using optimality theory and game theory (e.g., evolutionary stable strategy). ${ }^{11}$ These models show that the improvement of one trait that is a component of the fitness of an individual will have a cost on other fitness components. Traits that help to survive may have a cost in terms of fertility and/or reproduction (and vice versa).

In order to explain the evolution of age-structured populations, evolutionary biologists have therefore to take into account the trade-offs emerging from the different fitness components, which, in their turn, dictate the life span, senescence,

\footnotetext{
${ }^{9}$ Both equations make further simplifying assumptions: there is no transmission bias between parents and offspring, environmental conditions and population demography are constant, traits are normally distributed, etc. (Walsh and Lynch 2018).

10 More complex situations in age-structured populations may occur (for example, that there is no stable age distribution).

11 Lehtonen (2018) shows how to recover these different models using the Price equation.
} 
number and size of offspring, etc., of every organism in a population. ${ }^{12}$ To handle such scenarios, evolutionary biologists have combined different models from population genetics and population ecology. Traditionally-as we already mentioned-these models keep track of the evolutionary change using genetic and demographic parameters and adopting typical simplifying assumptions (such as our population being large enough to ignore stochastic effects, fecundity of females of a given age is independent of the age of their mates, etc.). More recently, age-structured versions of the breeder's and the Lande equations were derived for cases of weak selection and stable age structure (see Charlesworth, 1994 for a detailed analysis). In 2008, Coulson and Tuljapurkar (2008) presented an age-structured Price equation which was able to handle a wide variety of scenarios without appealing to the simplifying assumptions of the previous models. In particular, they decomposed fitness into age-specific components, instead of considering it a scalar. This allowed them to study different processes that affect organisms and their traits during their life histories (i.e., the progress of these organisms throughout their lives). Coulson and Tuljapurkar provided two equivalent versions of their equation (we present just one):

$$
\begin{aligned}
\Delta \bar{Z}= & \sum_{a=1}^{\omega-1}[\Delta c(a, t)] \bar{Z}(a, t)-c(\omega, t) \bar{Z}(\omega, t) \\
& +\sum_{a=1}^{\omega-1}\left(\frac{c(a, t)}{\bar{W}(t)}\right)[\operatorname{Cov}(Z, S)(a, t)+\overline{S G}(a, t)] \\
& +\sum_{a=1}^{\omega}\left(\frac{c(a, t)}{\bar{W}(t)}\right)[\bar{R}(a, t) \bar{Z}(a, t)+\bar{R}(a, t) \bar{D}(a, t)+\operatorname{Cov}(D, R)(a, t)+\operatorname{Cov}(Z, R)(a, t)]
\end{aligned}
$$

where the change in the mean of the trait $\mathrm{Z}, \Delta \bar{Z}$, is equal to the contribution in $\bar{Z}$ due to ageing $\sum_{a=1}^{\omega-1}[\Delta c(a, t)] \bar{Z}(a, t)-c(\omega, t) \bar{Z}(\omega, t)$, plus the contribution of age-specific processes $\sum_{a=1}^{\omega-1}\left(\frac{c(a, t)}{\bar{W}(t)}\right)$, such as survival selection differential on $Z \operatorname{Cov}(Z, S)$, plus the mean phenotypic plasticity $\overline{S G}$, plus the mean age-specific recruitment $\bar{R}$ and mean trait value $\bar{Z}$, plus the mean age-specific recruitment $\bar{R}$ and the mean difference between offspring and parental trait value $\bar{D}$, plus the covariation between offspring size and offspring number $\operatorname{Cov}(D, R)$, plus the selection differential on recruitment $\operatorname{Cov}(Z, R)$. All the terms on the right-hand side of the equation are calculated across all individuals in age and time $(a, t)$.

This detailed decomposition helps us to understand the complex interactions of different biological processes that act upon populations and makes it easier to find equilibrium situations. In the same way that the basic Price equation envisages two possible states of equilibrium (either both terms, the covariance and the expectation, are zero or they balance each other out), all these new terms in the age-structured Price equation show us a larger number of combinations by which a population may be in equilibrium. The most usual example of equilibrium is

$\overline{12}$ Also, phylogenetic constraints may produce additional trade-offs (Futuyma 2013). 
due to a trade-off between survival selection and fertility selection. Using the age-structured Price equation we can see that this condition is reached if there is zero transmission bias and stable demography, and selective pressures in survival and fertility balance each other out. Coulson and Tuljapurkar expressed this trade-off, formally, as

$$
\sum_{a=1}^{A} c(a+1) \frac{\operatorname{Cov}(Z, S)(a)}{\bar{S}}=-\sum_{a=1}^{\omega-1} c(a+1) \frac{\operatorname{Cov}(Z, R)(a)}{\bar{S}}\left(\frac{c(a, t)}{\bar{W}(t)}\right)
$$

where the left-hand side represents the effect of survival selection, and the righthand side represents the effect of fertility selection. Coulson and Tuljapurkar also described how to achieve other types of equilibrium involving the other forces acting upon populations, ${ }^{13}$ and applied their formalism to a specific age-structured population of red deer, finding no change in the mean trait even when survival selection acts strongly as individuals aged. This is due to the existence of substantial transmission biases, producing significant differences between parents' and offspring's trait values, and then balancing the action of survival selection.

This case study shows how researchers can put into practice the Pricean approach outlined in the previous section. Coulson and Tuljapurkar started with the basic ingredients of evolutionary systems described by Price and postulated new assumptions. They then derived a Price-like equation for age-structured populations. Next, they asked how many equilibrium states could be derived from this equation. Finally, they applied their age-structured Price equation to a real population. ${ }^{14}$

\subsection{Price-like equations outside traditional population genetics}

In the previous subsection, we discussed how the Price equation has been satisfactorily employed, outside traditional population genetics, to model evolutionary dynamics related to evolutionary ecology. This served us as an example to point out a more general tendency in evolutionary biology. Evolutionary biologists have adopted Price-like formalisms to account for a broad variety of evolutionary phenomena. Take, for instance, the recent studies on different mechanisms of inheritance beyond genetic inheritance, such as epigenetic, behavioural, or symbolic inheritance. Day and Bonduriansky (2011) applied the Pricean approach and developed the following equations for genetic $(g)$ and nongenetic $(h)$ inheritance

\footnotetext{
13 Of course, equilibrium can be also reached by selection alone (for example, survival selection), acting at different ages in opposite directions.

14 There already exist different versions of the Price equation dealing with different kinds of class-structured populations (Barfield et al., 2011; Grafen 2015). The age-structured Price equation does not take into account possible stochastic factors, such as demographic and environmental stochasticity (producing drift and fluctuating selection, respectively) that can affect the evolutionary trajectory of our target systems. Still, stochastic versions of the Price equation have been developed in order to handle this possibility (Rice 2008; Rice and Papadopoulos 2009; Engen and Saether 2014), by adding new assumptions (e.g., fitness is considered a random variable instead of a parameter).
} 
Table 1 Several mechanisms and phenomena (some of them vindicated by the Extended Evolutionary Synthesis) within the Price equation framework

$\bar{w} \Delta \bar{g}=\operatorname{Cov}(w, g)+E\left(b \Delta g^{b}\right)+E\left(p \Delta g^{p}\right)$

$\bar{w} \Delta \bar{h}=\operatorname{Cov}(w, h)+E\left(b \Delta h^{b}\right)+E\left(p \Delta h^{p}\right)$

Genetic and nongenetic Price equations (Day and Bonduransky, 2011)

$\Delta \bar{Z}=\frac{1}{\beta \bar{Z}+R}\left(\beta+\lambda_{2}\right) \sigma_{A}^{2}$

Niche construction (Otsuka, 2015)

$\dot{\bar{x}}^{A}=\operatorname{cov}_{A}\left(x, r^{A A}\right)-\mu\left(\bar{x}^{A}-\bar{x}_{m}^{A}\right)+\frac{N_{T}^{B}}{N_{T}^{A}} \operatorname{cov}_{B}\left(x, r^{B A}\right)+\frac{N_{T}^{B}}{N_{T}^{A}} r^{-B A}\left(\bar{x}^{A}-\bar{x}^{B}\right)$

The Price equation for multiple habitats (Day \& Gandon, 2006)

$\Delta \bar{z}=\frac{\operatorname{cov}\left(C_{*}^{a} z^{a}\right)}{\left(C_{*}^{*} / n^{a}\right)}-\frac{\operatorname{cov}\left(C_{d}^{*}, z_{d}\right)}{\left(C_{*}^{*} / n_{d}\right)}+\operatorname{ave}\left((\Delta z)_{j}^{i}\right)$

Macroevolutionary Price equation (Rankin et al., 2015)

$\widehat{\Delta \bar{\phi}}=\operatorname{cov}(\varphi, \hat{\Omega})+\operatorname{cov}(\widehat{\delta}, \hat{\Omega})+\overline{\operatorname{cov}_{i}(\widehat{\delta}, \Omega)}+\operatorname{cov}\left(\varphi_{p}, \hat{\Omega}_{p}\right)+\bar{\delta}_{p}$

Hierarchical Stochastic Price equation (Simpson, 2011)

$\Delta \bar{z}=\operatorname{cov}_{I}\left(w_{i}, z_{i}^{\prime}\right)+E_{I}\left[\operatorname{cov}_{J i}\left(w_{i j}, z_{i j}^{\prime}\right)\right]+E_{I}\left[E_{J i}\left[\Delta z_{i j}\right]\right]$

Community Price equation (Govaert et al., 2016)

$\Delta \bar{z}=\operatorname{Cov}(c, z)+E_{c}(\Delta z)$

Cultural Price equation (El Mouden et al., 2014)

$\phi^{2}=\frac{E\left(\Omega^{\Delta \star}\right) \Xi_{T}}{E\left(\mathrm{~V}^{\Delta \star}\right), E\left(\Omega^{\Delta \star}\right)_{T}}$

Network Price equation (Dragicevic, 2016)

$\frac{d \bar{z}}{d t}=\operatorname{Cov}(m, z)+E\left(\frac{d z}{d t}\right)$

Symbiosis Price equation (Smith, 2007)

$\Delta \operatorname{Var}(z)=\operatorname{Var}\left(\omega_{i}\right) E\left[\left(z_{p i}+\Delta z_{i}\right)^{2}\right]+\operatorname{Var}\left(\Delta z_{i}\right)$

ZFEL Price equation (Fleming, 2012)

$\Delta Q=\frac{\operatorname{Cov}(z, q)}{\bar{z}}+\sum_{j=1}^{k} \frac{\sum z_{i} \Delta q_{i j}}{N \bar{z}}$

Evolutionary algorithm Price equation (Basset et al., 2005)

$$
\begin{aligned}
& \bar{w} \Delta \bar{g}=\operatorname{Cov}(w, g)+E\left(b \Delta g^{b}\right)+E\left(p \Delta g^{p}\right) \\
& \bar{w} \Delta \bar{h}=\operatorname{Cov}(w, h)+E\left(b \Delta h^{b}\right)+E\left(p \Delta h^{p}\right)
\end{aligned}
$$

where the first terms of each equation represent the effect of natural selection on the genetic and nongenetic components; the second terms represent the "reproductive transmission", that is, the change in genetic and nongenetic values that occur during transmission from parent to offspring; and the third terms represent the "survival transmission", i.e., the change in genetic and nongenetic values that occur in parental individuals as they survive from one time step to the next. From these equations Day and Bonduriansky were able to derive specific models that represent concrete nongenetic mechanisms of inheritance (such as transgenerational epigenetic inheritance, maternal effects, environmental noise, or RNA inheritance).

This is an example of how the Pricean approach can account for many phenomena and mechanisms whose importance is stressed by the Extended Evolutionary 
Synthesis supporters (Lewens, 2019 makes a similar claim). As a matter of fact, besides nongenetic inheritance, this approach has already been applied to phenomena such as niche construction, hierarchies, macroevolution, or networks (see Table 1 for further examples). More generally, the Pricean approach is also able to account for many other evolutionary processes historically neglected or unnoticed by the Modern Synthesis that are not explicitly vindicated by the Extended Evolutionary Synthesis, such as symbiosis (e.g., symbionts transmissions, Smith, 2007), host-pathogen interactions (e.g., pathogens habitats, Day \& Gandon, 2006), or other processes that increase "pure complexity" (McShea \& Brandon, 2010) ${ }^{15}$ (see Table 1). ${ }^{16}$

These examples show how the logic and language of the Price equation can be used in several areas of evolutionary biology through the derivation of new forms of the Price equation. As this is not an algorithmic process, different researchers may develop different forms of the Price equation in a particular scientific area, depending on their assumptions and mathematical abilities. This, in its turn, may produce theoretical controversies. For instance, Aguilar and Akçay (2018) point out that a possible limitation of the general framework for genetic and nongenetic inheritance put forward by Day and Bonduriansky is that it provides distinct measures of fitness depending on the systems of inheritance. By contrast, Aguilar and Akçay argue that a single measure for genetic and nongenetic inheritance is necessary in order to elaborate reliable causal explanations. Thus, they develop their own Price-like equation for genetic and cultural inheritance incorporating both systems of inheritance and their fitness measures directly. ${ }^{17}$ Rather than being problematic, these theoretical controversies reflect an additional valuable characteristic of the Pricean approach: it demands clarity from modellers (about the entities and characters involved in the evolutionary process, their relationships and partitions, the time step, etc.) and thus facilitates the comparison and discussion of different formalisms.

\section{Evolutionary biology as a theoretical holon}

In Sect. 2, we observed that, when certain supporters of the fragmentalist view complain about the "physics envy" of some evolutionary biologists, they usually assume a picture of physical theories as structurally well-defined and all encompassing. Still,

\footnotetext{
15 The "pure complexity" of an organism is defined as "the amount of differentiation among its parts or, where variation is discontinuous, the number of part types" (McShea and Brandon 2010, p. 45). In order to explain the increase of organisms' pure complexity, McShea and Brandon argue that there is a background condition in all evolutionary systems. They call this condition the zero-force law of evolution (ZFEL): "In any evolutionary system in which there is variation and heredity, in the absence of natural selection, other forces, and constraints acting on diversity and complexity, diversity and complexity will increase on average" (McShea and Brandon 2010, p. 3). Fleming (2012) derived a mathematical expression of the ZFEL, based on the Price equation (see Table 1).

16 See also Rice 2004, and Luque 2017, for further evidence of the many applications of the Price equation.

17 See Baravalle and Luque (forthcoming) for a detailed analysis on Aguilar and Akçay's formalism and cultural evolutionary theory in general.
} 
while it can be argued that, as theory-nets, they can indeed be orderly reconstructedlike, we have argued in Sect. 3, the genetic theory of evolution as well -, this is not the whole story. Theory-nets do not live in a "theoretical vacuum", but they are surrounded by other theory-nets and models that complement them in crucial ways. Without these other theories and models - which are not directly derived from the original theorynet-no physical theory would be able to account for all the phenomena that the disciplinary field is called to explain. In other words, the explanation of a certain disciplinary domain of phenomena is never the result of a single theory (i.e., a theory-net) but, more broadly, of a "net of theory-nets", that is, a theoretical holon.

In non-relativistic classical mechanics, for instance, it is fair to acknowledge that Newtonian mechanics, while certainly central in the overall understanding of the phenomena related to physical motion, has been historically complemented by many other auxiliary theories. These theories_-such as celestial mechanics, particle mechanics, aero- and hydro-dynamics, or the kinetic theory of gases, among others (Corben \& Stehle, 1994) — are fundamental in explaining specific physical phenomena. Even though they are conceptually dependent on Newtonian mechanics, their characteristic laws are not, strictly speaking, specialisations of Newton's second law. In order to account for the phenomena that they are called on to explain, the formulation of these theories required a modification of Newton's second law.

For instance, from the original formulation of classical mechanics, physicists recognised that Newton's second law, as originally stated, was not able to account for rotational motion (Sklar, 2013). This was because Newton's second law employed the notion of "inertial mass" (i.e., bodies' resistance to change of their linear motion). However, in order to explain rotational motion, an additional type of inertia-i.e., bodies' resistance to change of their rotational motion-must be taken into account. Thus, the law of conservation of linear momentum needed to be supplemented by a law of conservation of angular momentum. This new law made it necessary to invent the concept of "moment of inertia". In addition, new kinds of forces-i.e., rotational forces or "torques"-were introduced to supplement the traditional linear forces. Thus, the following equation, analogous to Newton's original second law, was developed

$$
\sum \tau=I \alpha
$$

where $\tau$ represents a torque, $I$ the moment of inertia, and $\alpha$ the angular momentum. Here, the work of Euler was essential, which is why this equation is sometimes called Euler's second law. It was subsequently applied to the rotation of rigid bodies, and this application led to the formulation of Euler's equations of motion for rigid body dynamics.

In a similar fashion, fluid mechanics had to wait until the work of Cauchy in order to gain its fundamental equation (Granger, 1995). The following equation is known as the Cauchy momentum equation,

$$
\rho \frac{D u}{D t}=\nabla \cdot \sigma+F
$$

where $\rho$ is the density of the fluid, $u$ represents flow velocity (therefore $\frac{D u}{D t}$ is the acceleration of the flow), $\nabla$ is the gradient, $\sigma$ is the stress tensor, and $F$ represents the 
body forces acting on the fluid. This equation is valid for any kind of fluid and takes specific forms depending on the characteristics of the fluid (viscosity, compressibility, etc.), yielding the Navier-Stokes equations, Stokes' flow equation, Euler's equations for fluid dynamics, or the Stokes laws for friction and sound attenuation.

However, we may possibly find the most striking example in Langevin dynamics. Coinciding with the two major physics revolutions of the twentieth century (relativity and quantum mechanics), Paul Langevin extended the Newtonian approach to stochastic systems. Langevin dynamics studies the dynamics of molecular systems. The first phenomenon to which Langevin applied his new approach was Brownian motion. This is stochastically modelled by the following Langevin equation ${ }^{18}$ :

$$
m \frac{d^{2} x}{d t^{2}}=-6 \pi \mu a \frac{d x}{d t}+X
$$

where $x$ represents the location of the particle within the fluid, $m$ is its mass, $-6 \pi \mu a$ characterises the damping coefficient (i.e., fluid viscosity that slows the movement of the particles), and $X$ denotes a random "noise term" which describes the effect of the collisions between fluid atoms or molecules. In the first English translation of Langevin's 1908 work, Lemons observes: “Langevin applied Newton's second law to a representative Brownian particle. In this way Langevin invented the ' $\mathrm{F}=\mathrm{ma}$ ' of stochastic physics now called the 'Langevin equation'", (Lemons 1997, p. 1079). This is a nice example of how Newton's second law can be a fruitful inspiration to model and explain forces-i.e., stochastic forces-that could not be explained within the deterministic framework of Newtonian mechanics.

All these examples show that, even in the limited context of non-relativistic classical mechanics, Newton's second law is not a "catch-all" equation. In order to guarantee a progressive understanding of the physical world, it needed to be reformulated accordingly. Note, in this regard, that these reformulations of Newton's second law play, in classical mechanics, a role that is (at least prima facie) analogous to that developed by the Price-like equations that we considered in the previous sections. In both cases, these modified equations extend the applicability of concepts originally conceived to model certain phenomena to other related phenomena initially neglected.

One may think of the reformulations of Newton's second law as new fundamental laws for new theory-nets that complemented the original theory-net of Newtonian mechanics. As a matter of fact, structuralists have already worked out the details of the theory-nets of some of these other theories (e.g., particle mechanics; Balzer et al., 1987). What we would like to stress here, nonetheless, is not the fact that each theory can be depicted as a new theory-net. Instead, we would like to point out that, at the level of the theoretical holon, classical mechanics does not exhibit a rigidly hierarchical structure. It is rather a "family" of theory-nets, which exchange concepts and models that holistically account for the physical world.

\footnotetext{
${ }_{18}$ The general Langevin equation is of the form: $\dot{q}=F(q)+\sqrt{Q} \xi(t)$, where $F(q)$ represents deterministic forces, and $\sqrt{Q} \xi(t)$ is the stochastic term (i.e., additive white noise with a zero mean and a Gaussian distribution) (Fuchs 2013).
} 
We can imagine the Price equation as a heuristic device for all the theoretical projects, such as those we discussed in Sect. 4 (e.g., the study of structured populations in evolutionary ecology, the analysis of non-genetic channels of inheritance, macroevolutionary theory, hierarchy theory, etc.), that aim to be considered as evolutionary. These theoretical projects compose what we may conceive as the theoretical holon of evolutionary biology. While in its original form the equation is indeed neutral with regard to the specificities of any particular evolutionary process, further modifications of the equation allow the capture of the salient traits of different evolutionary systems. In this sense, the theoretical approach based on the Price equation in evolutionary biology is not different from the approach that physicists have adopted to build theories from Newton onward. Starting from a general assumption concerning the overall features of the phenomena under study, Newton's followers worked on progressively more specific applications of Newton's second law, enriching Newtonian mechanics with the details that made it such a successful theory. The concepts elaborated within Newtonian mechanics were then exported to other physical domains, and modified according to the needs and interests of the practitioners. The Price equation, similarly, has first allowed a systematisation of the factors assumed to act in population genetics, and it is progressively being adopted in other areas of evolutionary biology as a framework for explaining other dynamical processes.

Possibly not every phenomenon traditionally accounted for by evolutionary biology perfectly fits the explanatory schema suggested by the Price equation. Moreover, of course, the Pricean approach to evolution is still too young to observe the constitution of well-defined theory-nets complementary to the genetic theory of evolution, analogous to rigid-body dynamics and fluid dynamics for classical mechanics (we should not forget that these complementary theories are the fruit of centuries of intellectual effort). However, we think (relying on the evidence we presented in Sects. 3 and 4 ) that there is room for optimism concerning the formulation of a general theoretical framework of evolution resembling those that we find in physics.

\section{Conclusions}

In this article we have argued, against a quite popular view in the philosophy of biology, that notwithstanding the complexity of evolutionary dynamics, we should not be pessimistic about the possibility of attaining an encompassing mathematical theory of evolution. The core of this theory should be the Price equation. In order to support this claim, we first grounded, in Sect. 2, our argument on a sophisticated conception of the structure of scientific theories (i.e., metatheoretical structuralism). This allowed us to spell out a useful distinction between theory-nets and theoretical holons, as well as to dissipate some common misunderstandings about the role of different kinds of laws in scientific theories. From this, in Sect. 3, we outlined the structure of the genetic theory of evolution (aka traditional population genetics) and clarified the fundamental role that the Price equation plays in it. In Sect. 4 we broadened our scope, by showing how a Pricean approach to evolution is also being successfully adopted outside the boundaries of traditional population genetics in order 
to account for a variety of evolutionary phenomena. Finally, in Sect. 5, we compared the possible development of evolutionary biology with the historical development of classical mechanics. Newtonian mechanics fully disclosed its explanatory power to the domain of all physical phenomena through a continuous reformulation and adaptation of Newton's second law. Analogously, we may expect-relying on the current advances in evolutionary biology - that a Pricean framework can help to coordinate the theoretical efforts of the practitioners.

In this sense, our characterisation of evolutionary theory as a holon, with the Price equation at its core, reinforces the ideas of unification and generality. We recognise the plural nature of evolutionary phenomena and of its research, as well as the necessity to bring together as different and complementary approaches, models, methodologies, and theories as possible, acknowledging their scope and limitations (Griesemer, 2006; Mitchell \& Dietrich, 2006). The Pricean approach does not restrict our view of evolution to a unique theory or model. In order to allow us to explain specific phenomena, the Price equation can be complemented by further specific modelling techniques (including several causal modelling techniques) and additional information about the target system (Frank \& Fox, 2020; Morrissey, 2014; Otsuka, 2016). However, what we wanted to stress is that all these different models, techniques, and methods can be connected under the formal umbrella provided by the Price equation, which may help researchers to develop bona fide scientific evolutionary theories, therefore giving us a sense of unification (Luque, 2017). Thus, the Price equation can be seen as the bridge between different approaches and different theories, encompassing the complex evolutionary world through its austere and elegant formalism.

Acknowledgements We are thankful to two anonymous reviewers for their comments on an earlier version of the manuscript. VL was supported by the Spanish Ministry of Science, Innovation and Universities under Juan de la Cierva fellowship (FJC2018-038661-I), and Projects FFI2016-76799-P and FFI2017-89639-P. LB would like to thank the Fundação para a Ciência e a Tecnologia de Portugal (FCT Contract No. DL57/2016/CP1479/CT0064) for the financial support.

Funding VL was supported by the Spanish Ministry of Science, Innovation and Universities under Juan de la Cierva fellowship (FJC2018-038661-I), and Projects FFI2016-76799-P and FFI2017-89639-P. LB would like to thank the Fundação para a Ciência e a Tecnologia de Portugal (FCT Contract No. DL57/2016/CP1479/CT0064) for the financial support.

\section{Declarations}

Conflict of interest The authors declare that they have no conflict of interest.

Open Access This article is licensed under a Creative Commons Attribution 4.0 International License, which permits use, sharing, adaptation, distribution and reproduction in any medium or format, as long as you give appropriate credit to the original author(s) and the source, provide a link to the Creative Commons licence, and indicate if changes were made. The images or other third party material in this article are included in the article's Creative Commons licence, unless indicated otherwise in a credit line to the material. If material is not included in the article's Creative Commons licence and your intended use is not permitted by statutory regulation or exceeds the permitted use, you will need to obtain permission directly from the copyright holder. To view a copy of this licence, visit http://creativecommons.org/licen ses/by/4.0/. 


\section{References}

Aguilar, E., \& Akçay, E. (2018). Gene-culture co-inheritance of a behavioral trait. The American Naturalist, 192(3), 311-320.

Balzer, W., Moulines, C. U., \& Sneed, J. D. (1987). An Architectonic for Science. The Structuralist Program. Reidel.

Baravalle, L. \& Luque, V. J. (forthcoming) Towards a Pricean foundation for cultural evolutionary theory. Theoria.

Baravalle, L., \& Vecchi, D. (2020). Drift as a force of evolution: A manipulationist account. In L. Baravalle \& L. Zaterka (Eds.), Life and evolution: Latin American essays on the history and philosophy of biology (pp. 143-162). Springer.

Barfield, M., Holt, R., \& Gomulkiewicz, R. (2011). Evolution in stage-structured populations. The American Naturalist, 177(4), 397-409.

Bartholomew, G. (1986). The role of natural history in contemporary biology. BioScience, 36(5), 324-329.

Bassett, J., Potter, M. \& De Jong, K. (2005). Applying Price's Equation to Survival Selection. In GECCO, pp $1-8$.

Beatty, J. (1995). The evolutionary contingency thesis. In G. Wolters \& J. G. Lennox (Eds.), Concepts, theories, and rationality in the biological sciences (pp. 45-81). Universitätsverlag.

Carnap, R. (1995). An introduction to the philosophy of science. Dover Publications.

Charlesworth, B. (1994). Evolution in age-structured populations (2nd ed.). Cambridge University Press.

Corben, H. C., \& Stehle, P. (1994). Classical mechanics (2nd ed.). Dover Publications.

Coulson, T., \& Tuljapurkar, S. (2008). The dynamics of a quantitative trait in an age-structured population living in a variable environment. The American Naturalist, 172, 599-612.

Day, T., \& Bonduriansky, R. (2011). A unified approach to the evolutionary consequences of genetic and nongenetic inheritance. The American Naturalist, 178, E18-E36.

Day, T., \& Gandon, S. (2006). Insights from Price's equation into evolutionary epidemiology. In Z. Feng, U. Dieckmann, \& S. Levin (Eds.), Disease evolution: Models, concepts, and data analysis (pp. 23-44). American Mathematical Society.

Depew, D. J., \& Weber, H. (1996). Darwinism evolving: System dynamics and the genealogy of natural selection. The MIT Press.

Diederich, W., Ibarra, A., \& Mormann, T. (1994). Bibliography of structuralism (1989-1994 and additions). Erkenntnis, 40, 403-418.

Díez, J., \& Lorenzano, P. (2002). Desarrollos actuales de la metateoría estructuralista. Universidad Nacional de Quilmes.

Díez, J., \& Lorenzano, P. (2013). Who got what wrong? Fodor and Piattelli on Darwin: Guiding principles and explanatory models in natural selection. Erkenntnis, 78, 1143-1175.

Dragicevic, A. (2016). From robustness to resilience: A network Price identity approach. Ecological Complexity, 28, 47-53.

Egler, F. E. (1986). 'Physics envy' in ecology. Bulletin of the Ecological Society of America, 67, $233-235$.

El Mouden, C., André, J.-B., Morin, O., \& Nettle, D. (2014). Cultural transmission and the evolution of human behaviour: A general approach based on the Price equation. Journal of Evolutionary Biology, 27, 231-241.

Engen, S., \& Saether, B. E. (2014). Evolution in fluctuating environments: Decomposing selection into additive components of the Robertson-Price equation. Evolution, 68(3), 854-865.

Feigl, H. (1970). The "orthodox" view of theories: remarks in defence as well as critique. University of Minnesota Press, Minneapolis. Retrieved from the University of Minnesota Digital Conservancy, http://hdl.handle.net/11299/184651.

Fisher, R. (1932). The evolutionary modification of genetic phenomena. In Proceedings of the 6th International Congress of Genetics, 1, pp. 165-172.

Fleming, L. (2012). Variance, Selection and evolutionary explanation. PhD Dissertation Thesis. Duke University.

Fodor, J., \& Piattelli-Palmarini, M. (2010). What Darwin got wrong. Profile Books.

Frank, S. A. (1986). Dispersal polymorphism in subdivided populations. Journal of Theoretical Biology, 122, 303-309.

Frank, S. A. (1995). George Price's contributions to evolutionary genetics. Journal of Theoretical Biology, 175, 373-388. 
Frank, S. A. (2012). Natural selection. IV The Price equation. Journal of Evolutionary Biology, 25, 1002-1019.

Frank, S. A., \& Fox, G. A. (2020). The inductive theory of natural selection. In S. M. Scheiner \& D. P. Mindell (Eds.), The theory of evolution (pp. 171-193). University of Chicago Press.

Frank, S. A., \& Slatkin, M. (1990). The distribution of allelic effects under mutation and selection. Genetical Research, 55, 111-117.

Friedman, M. (2001). Dynamics of reason. CSLI Publications.

Fuchs, A. (2013). Nonlinear dynamics in complex systems. Springer.

Futuyma, D. (2013). Evolution (3rd ed.). Sinnauer.

Gardner, A. (2011). Kin selection under blending inheritance. Journal of Theoretical Biology, 284, $125-129$.

Ginnobili, S. (2018). La teoría de la selección natural. Bernal (Argentina), Universidad Nacional de Quilmes.

Godfrey-Smith, P. (2009). Darwinian populations and natural selection. Oxford University Press.

Govaert, L., Pantel, J., \& De Meester, L. (2016). Eco-evolutionary partitioning metrics: Assessing the importance of ecological and evolutionary contributions to population and community change. Ecology Letters, 19(8), 839-853.

Grafen, A. (2015). Biological fitness and the Price equation in class-structured populations. Journal of Theoretical Biology, 373, 62-72.

Granger, R. (1995). Fluid mechanics. Dover Publications.

Griesemer, J. (2006). Theoretical integration, cooperation, and theories as tracking devices. Biological Theory, 1(1), 4-7.

Hempel, C. G. (1965). Aspects of scientific explanation and other essays in the philosophy of science. The Free Press.

Hitchcock, Ch., \& Velasco, J. (2014). Evolutionary and Newtonian forces. Ergo, 1, 39-77.

Jammer, M. (1956). Concepts of force: A study in the foundations of dynamics. Dover.

Kerr, B., \& Godfrey-Smith, P. (2009). Generalization of the Price equation for evolutionary change. Evolution, 63, 531-536.

Kitcher, P. (1989). Explanatory unification and the causal structure of the world. In P. Kitcher \& W. C. Salmon (Eds.), Scientific explanation (pp. 410-505). Minneapolis.

Laland, K. N., Uller, T., Feldman, M. W., Sterelny, K., Müller, G. B., Moczek, A., Jablonka, E., \& Odling-Smee, J. (2015). The extended evolutionary synthesis: Its structure, assumptions and predictions. Proceedings of the Royal Society B, 282, 20151019.

Lehtonen, J. (2018). The Price equation, gradient dynamics, and continuous trait game theory. The American Naturalist, 191, 146-153.

Lehtonen, J. (2020). The Price equation and the unity of social evolution theory. Philosophical Transactions of the Royal Society B, 375, 20190362.

Lemons, D. \& Gythiel, A. (1997). Paul Langevin's 1908 paper "on the theory of brownian motion" ["Sur la théorie du mouvement brownien," C. R. Acad. Sci. (Paris) 146, 530-533 (1908)]. American Journal of Physics 65(11), 1079-1081.

Lewens, T. (2019). The extended evolutionary synthesis: What is the debate about, and what might success for the extenders look like? Biological Journal of the Linnean Society, 127, 707-721.

Lockwood, D. R. (2007). Ecology is not rocket science. Emergence Complexity and Organization, 9(2), 49-58.

Lorenzano, P. (2006). Fundamental laws and laws of biology. In: Ernst, G. \& Niebergall, K. G. (Ed.). Philosophie derWissenschaft - Wissenschaft der Philosophie. Festschrift für C. Ulises Moulines zum 60. Geburstag. Paderborn:Mentis-Verlag, 2006. pp. 129-55

Lorenzano, P. (2014). What is the status of the Hardy-Weinberg law within population genetics? In M. C. Galavotti, E. Nemeth, \& F. Stadler (Eds.), European philosophy of science - philosophy of science in Europe and the Viennese heritage. Vienna circle institute yearbook. (Vol. 17). Springer.

Lorenzano, P., \& Díaz, M. A. (2020). Laws, models, and theories in biology: A unifying interpretation. In L. Baravalle \& L. Zaterka (Eds.), Life and Evolution: Latin American essays on the history and philosophy of biology (pp. 163-207). Springer.

Luque, V. J. (2017). One equation to rule them all: A philosophical analysis of the Price equation. Biology and Philosophy, 32(1), 97-125.

Marshall, J. A. R. (2015). Social evolution and inclusive fitness theory: An introduction. Princeton University Press.

Mayr, E. (2004). What makes biology unique. Cambridge University Press. 
McShea, D., \& Brandon, R. (2010). Biology's first law: The tendency for diversity and complexity to increase in evolutionary systems. The University of Chicago Press.

Mitchell, S., \& Dietrich, M. (2006). Integration without unification: An argument for pluralism in the biological sciences. The American Naturalist, 168(S6), S73-S79.

Morrissey, M. (2014). Selection and evolution of causally covarying traits. Evolution, 68-6, 1748-1761.

Moulines, C. U. (1984a). Existential quantifiers and guiding principles in physical theories. In J. J. E. Gracia, E. Rabossi, E. Villanueva, \& M. Dascal (Eds.), Philosophical Analysis in Latin America (pp. 173-198). Reidel.

Moulines, C. U. (1984b). Links, loops, and the global structure of science. Philosophia Naturalis, 24, $254-265$.

Moulines, C. U. (1991). Pluralidad y recursión. Estudios epistemológicos. Alianza editorial.

Moulines, C. U. (2010). Metatheoretical structuralism: A general program for analyzing science. Axiomathes, 20, 255-268.

Nowak, M., \& Highfield, R. (2011). Supercooperators: Altruism, evolution, and why we need each other to succeed. Free Press.

O'Hara, R. (2005). The anarchist's guide to ecological theory. Or, we don't need no stinkin' laws. Oikos, $110(2), 390-393$.

Otsuka, J. (2015). Using causal models to integrate proximate and ultimate causation. Biology and Philosophy, 30(1), 19-37.

Otsuka, J. (2016). Causal foundations of evolutionary genetics. The British Journal for the Philosophy of Science, 67(1), 247-269.

Pigliucci, M. (2002). Are ecology and evolutionary biology "soft" sciences? Ann. Zool. Fennici, 39, 87-98.

Pigliucci, M., \& Müller, G. B. (Eds.). (2010). Evolution, the extended synthesis. MIT Press.

Popper, K. R. (1974). Darwinism as a metaphysical research program. In P. Schilpp (Ed.), The philosophy of Karl Popper (IL), Open court (pp. 133-143). La Salle.

Price, G. R. (1970). Selection and covariance. Nature, 227, 520-521.

Price, G. R. (1972). Extension of covariance selection mathematics. Annals of Human Genetics, 35, 485-490.

Queller, D. (2017). Fundamental theorems of evolution. American Naturalist, 189(4), 345-353.

Rankin, B. D., Fox, J. W., Barrón-Ortiz, C. R., Chew, A. E., Holroyd, P. A., Ludtke, J. A., Yang, X., \& Theodor, J. M. (2015). The extended Price equation quantifies species selection on mammalian body size across the palaeocene/eocene thermal maximum. Proceedings of the Royal Society B, 282, 20151097.

Rice, S. H. (2004). Evolutionary theory: Mathematical and conceptual foundations. Sinauer Associates.

Rice, S. H. (2008). A stochastic version of the Price equation reveals the interplay of deterministic and stochastic processes in evolution. BMC Evolutionary Biology, 8, 262.

Rice, S. H. (2020). Universal rules for the interaction of selection and transmission in evolution. Philosophical Transactions on Royal Society B, 375, 20190353.

Rice, S. H., \& Papadopoulos, A. (2009). Evolution with stochastic fitness and stochastic migration. PLoS ONE, 4, e7130.

Simpson, C. (2011). How many levels are there? How insights from evolutionary transitions in individuality help measure the hierarchical complexity of life. In B. Calcott \& K. Sterelny (Eds.), The major transitions in evolution revisited (pp. 199-226). The MIT Press.

Sklar, L. (2013). Philosophy and the foundations of dynamics. Cambridge University Press.

Smith, J. (2007). A gene's-eye view of symbiont transmission. American Naturalist, 170, 542-550.

Tawfik, D. (2010). Messy biology and the origins of evolutionary innovations. Nature Chemical Biology, 6(10), 692-696.

van Veelen, M. (2005). On the use of the Price equation. Journal of Theoretical Biology, 237, 412-426.

Walsh, B., \& Lynch, M. (2018). Evolution and selection of quantitative traits. Oxford University Press.

Waters, C. K. (2011). Okasha's unintended argument for toolbox theorizing. Philosophy and Phenomenological Research, 82(1), 232-240.

Publisher's Note Springer Nature remains neutral with regard to jurisdictional claims in published maps and institutional affiliations. 